\title{
Traumasensible Ansätze bei mediatisierter ritueller Gewalt: Constantin
}

„Ist es ein ritueller Kontext, bedarf es einer anderen Art des Arbeitens." (Traumafachberaterin, spezialisierte Fachberatung rituelle Gewalt, Focus Group Interviews).

- Zusammenfassung Mediatisierte sexualisierte Gewalt wird auch im Zusammenhang organisierter und ritueller Gewaltstrukturen eingesetzt, um Machtverhältnisse auszubauen, Betroffene an die Strukturen zu binden, sie mittels digitaler Medien zu kontrollieren und zu manipulieren. Die Belastungen der Betroffenen sind besonders komplex. Bisweilen leiden sie unter einer dissoziativen Identitätsstörung. Die Abhängigkeiten von den Gewaltstrukturen erschweren Hilfeprozesse. Die Wahrscheinlichkeit, dass weiterhin Kontakt zu den Strukturen besteht, muss auch nach einer Teilaufdeckung stets mitbedacht werden. Dieses Kapitel setzt sich aufgrund der Komplexität vor allem mit traumasensiblen Ansätzen der Arbeit mit von mediatisierter ritueller Gewalt betroffenen jungen Menschen auseinander. Es reflektiert dazu Möglichkeiten und Grenzen „gewaltfreier Räume“ für Adressat*innen. In diesem Zusammenhang wird auf die hervorgehobene Bedeutung der Psychohygiene für Helfende verwiesen.

\section{Fallvignette Constantin}

Die Sozialpädagogin einer betreuten Wohngruppe vermittelt den 15-jährigen Constantin an eine Fachberatungsstelle, die zu sexualisierter Gewalt arbeitet. Der Junge hat auf seinem Smartphone Videos gespeichert, die Sexualhandlungen mit scheinbar Minderjährigen sowie Folterszenen zeigen. Ein 
Problembewusstsein kann die Sozialpädagogin nicht erkennen. Er habe nur etwas „recherchiert“, so der Junge.

Aus Constantins Akte ist bekannt, dass er zwischen dem achten und dreizehnten Lebensjahr vom im Haushalt lebenden Partner der Kindesmutter, P., sexuell missbraucht wurde. Die Aufdeckung fand durch polizeiliche Ermittlungen statt. Bei mehreren Personen, darunter P., wurden tausende Missbrauchsaufzeichnungen sichergestellt. Constantin war darauf zu erkennen. Die Bestrafung P.s erfolgte ohne Anhörung Constantins.

In der Beratung thematisiert Constantin sein Verhältnis zu P. und wie sich ihre Unternehmungen, echte „Männersachen“, meistens um Technik drehten. P. habe eine Drohne mit einer hochauflösenden Kamera besessen. Mehrmals haben sie sich mit dem Tor-Netzwerk verbunden. Constantin wirkt auf den Berater fasziniert und stolz. Auf die Intervention des Beraters, was P. außerdem unternommen habe, um Constantin zu täuschen, wirkt der Junge erschrocken. Er flüstert: „Einen sicheren Freund erkennt man in unsicherer Sache." Constantin will wissen, ob der Berater glaube, dass P. sehr enttäuscht sei, wegen Constantin im Gefängnis zu sein und ob P. erfährt, was in der Beratung besprochen wird. Wiederholt nennt er ihn Prometheus.

Der Berater spiegelt Constantin: „Manchmal machen dir die Erinnerungen an P. immer noch ganz schön viel Angst.“ Der Junge blickt zu Boden. Wie zur Erklärung zeigt Constantin dem Berater einen Blogeintrag in einem GamingForum. „Ikarus_1.0: Körperlos. Sacke im schwarzen Lack. Eisige Tentakel. Pupillen im Interface. Platzangst. Im Auge der Blackbox. Wellen prallen. Echokammer. Du bist nicht einer. Du bist viele. Legion. An keinem Ort. In keiner Zeit scharren Klauen in der Dunkelheit. Ich rufe zu Dir. Puppet Master! Keine Antwort. Ghost. Büchse der Pandora. KI. Autonom.“

\section{Reflexionsfragen}

- Was löst die Notiz in uns aus?

- Wie stehen wir zu den Personen und ihren Handlungen?

- Wie kann die Situation fachlich eingeordnet werden? 


\subsection{Reflexionen zur fachlichen Einordnung des Fallbeispiels}

Die Vignette stellt eine komplexe Fallstruktur dar, deren wesentliche Zusammenhänge zunächst hervorgehoben werden:

1. Die Sozialpädagogin einer betreuten Wohngruppe nimmt sexualisierte Gewaltdarstellungen auf dem Smartphone eines 15-jährigen Adressaten zum Anlass, dem Jungen spezialisierte Hilfen zu vermitteln. Der Besitz der offensichtlichen Aufnahmen und das scheinbar fehlende Problembewusstsein Constantins stehen in einem Verhältnis zu dessen eigener Betroffenheit von strafrechtlich relevantem schwerem sexuellem Kindesmissbrauch durch ein Täter*innenNetzwerk bzw. eine Gruppe (,,bei mehreren Personen [...] wurden tausende Missbrauchsaufzeichnungen sichergestellt").

2. Im Beratungssetting verstärkt sich der Eindruck, dass Constantin weiterhin Abhängigkeiten gegenüber dem Haupttäter P. erlebt. Die Aussage „einen sicheren Freund erkennt man in unsicherer Sache“ weist auf einen Loyalitätskonflikt des Jungen hin und wirkt wie die Fortsetzung von Schweigegeboten. Damit verbundene Ängste drücken sich in der Erschrockenheit des Jungen sowie in der Sorge aus, der Täter könne erfahren, was in der Beratung besprochen wird.

3. Fallfakten (mehrere Täter*innen, schwerer sexueller Kindesmissbrauch, Herstellung und Besitz von Missbrauchsabbildungen) und Äußerungen Constantins, die auf eine Einbettung der Gewalt in ein Symbolsystem hinweisen, zum Beispiel Versatzstücke aus der griechischen und römischen Antike wie die Bezeichnungen „Prometheus“, „Ikarus“ und „Büchse der Pandora“, lösen in den Focus Group Interviews Assoziationen organisierter oder sogar ritueller Gewalt aus. Unter rituellen Gewaltstrukturen werden Täter*innenstrukturen mit (pseudo-) ideologischen, kultischen Hintergründen verstanden (Igney, 2019; Schröder et al., 2020), wobei Ideologie als Rechtfertigungsgrund für die Gewalt zu verstehen ist. Rituelle Gewalt verfolgt zumeist das Ziel, Machtstrukturen aufzubauen. Hierarchien und Unterwerfung sind daher oftmals symbolische Gegenstände der Ideologie. Zum Beispiel knüpft rituelle Gewalt an mystische, autoritäre Glaubenssysteme - etwa Satanismus, fundamentalistisches Christentum, Esoterik - oder Weltanschauungen - antike Mythologie, Päderastie, Faschismus - an (Fliß et al., 2016, 2018; Igney, 2012, 2019).

Die nachfolgenden Reflexionen wenden sich konzentriert den drei hervorgehobenen Aspekten a) zur Einschätzung des Funds von Missbrauchsabbildungen bei 
Constantin, b) Constantins Abhängigkeiten gegenüber P. und c) dem Komplex organisierter sowie ritueller Gewalt zu. Wir raten Professionellen, die in einem Fall rituelle Gewalt vermuten, selbst Unterstützung bei Expert*innen zu suchen. Wir verweisen auf das bundesweite berta-Telefon des Unabhängigen Beauftragten für Fragen des sexuellen Kindesmissbrauchs (UBSKM) in Kooperation mit N.I.N.A. e. V. (Nationale Infoline, Netzwerk und Anlaufstelle zu sexueller Gewalt an Mädchen und Jungen).

\title{
6.1.1 Einordnung des Besitzes von Missbrauchsabbildungen und Foltervideos
}

Der Besitz von Missbrauchsabbildungen und Foltervideos wird im Falle Constantins durch dessen eigene sexualisierte Missbrauchserfahrungen kontextualisiert. Der Besitz ist deshalb nicht unproblematisch. Je nach Art der Videos macht sich Constantin strafbar. Auch als Hinweis auf eine sich entwickelnde Übergriffigkeit oder Sexualdelinquenz ${ }^{1}$ sind der Besitz und vor allem mögliche Formen der Nutzung der Videos zu beachten. Zugleich sind mehrere Ursachenkonstellationen für den Besitz der Videos denkbar. In den Focus Group Interviews wird darauf verwiesen, dass Betroffene von mediatisierter Gewalt im Internet nach Abbildungen ihres eigenen Missbrauchs suchen, um Kontrolle und Handlungsmacht zurückzugewinnen.

\begin{abstract}
„Es könnte ja auch sein, dass er im Netz nach seinen eigenen Missbrauchsaufzeichnungen sucht. Und ein bisschen so Kontrolle, in Anführungsstrichen, darüber zu bekommen auch, ne. Das machen die ja dann auch häufig, ne, dass sie im Netz unterwegs sind, um zu gucken, da bin ich zu sehen und da bin ich zu sehen, ne.“
\end{abstract}

Kinder- und Jugendpsychotherapeutin, spezialisierte Fachberatung, FGI

Der Besitz der Videos kann Ausdruck einer subjektiven Bewältigungsstrategie Constantins sein, die ihn vorübergehend entlastet. Jedoch ist diese Strategie potenziell strafwürdig. Dafür fehlt Constantin augenscheinlich das Problembewusstsein. Zudem stellt sich die Frage, ob seine Bewältigungsstrategie nachhaltig wirksam ist, da er sich erneut mit Gewaltabbildungen konfrontiert. Der Nachhaltigkeit steht somit gegenüber, dass Constantins Belastungen reaktiviert werden oder er deviante Verhaltensweisen entwickelt. In der klinischen Psychologie

\footnotetext{
${ }^{1}$ Von Sexualdelinquenz sprechen wir im Falle gewohnheitsmäßiger, sexualisiert grenzverletzender Verhaltensweisen (Hanfland, 2011).
} 
wird zudem das Konzept des Self-Triggering, also der gezielten Selbstkonfrontation Betroffener mit retraumatisierenden Inhalten, diskutiert. Self-Triggering wird zumeist als maladaptives selbstverletzendes Verhalten gewertet (Swerdlow et al., 2020). Neuere Auseinandersetzungen mit dem Modell unterscheiden jedoch Motivationen des Self-Triggering, die von selbstverletzendem Verhalten und Selbstbestrafung bis zur Kontrolle über die Symptome einer posttraumatischen Belastungsstörung reichen. Zusammenfassend diene Self-Triggering dazu, ein konsistentes Weltbild wiederherzustellen, indem die nicht greifbare Bedeutung des traumatischen Erlebnisses mittels Wiedererleben und Kontrolle schwer steuerbarer Belastungsreaktionen in einen sinnvollen Zusammenhang zueinander gebracht werden solle (Bellet et al., 2020). Letzterer Ansatz liefert ungeachtet seiner klinischen Perspektivität wichtige Anregungen für die in den Handlungsempfehlungen aufgenommene Haltung einer systemischen Traumapädagogik, welche prinzipiell vom „guten Grund“ von Verhaltensweisen ihrer Adressat*innen ausgeht (Weiß, 2017). Weiterhin ist denkbar, dass Constantin die Videoaufnahmen nicht selbst gesucht hat, sondern sie dem ursprünglichen Gewaltkontext entstammen. Dem HUMAN-Projekt wurde ein solcher Fall dokumentiert.

„In diesem Fall geht es um die Vermutung auf innerfamiliären sexuellen Missbrauch
und rituelle Gewalt aufgrund eines Fundes von Missbrauchsabbildungen. Die Klientin
fand im Alter von [15-17] Jahren auf ihrem Rechner Dateien von Missbrauchsabbil-
dungen von sich selbst [...], die sie nicht zuordnen konnte. Die Fotos zeigten sie selbst
im Rahmen einer Gruppenvergewaltigung im Alter von etwa sechs bis zehn Jahren.“

Falldokumentation 6.1

Augenscheinlich ergibt sich eine Diskrepanz zwischen der Hypothese, auch in Constantins Fall könnten die Videos dem ursprünglichen Kontext entstammen und Constantins Erklärung, er habe recherchiert. Trotzdem ist nicht auszuschließen, dass die Videos auf fortbestehende Täter*innenkontakte hinweisen. Im Verhältnis zu Constantins Abhängigkeiten und Ängsten wehrt er möglicherweise mit der Behauptung, die Aufnahmen selbst gesucht zu haben, dahingehende Hinweise ab.

\subsubsection{Abhängigkeitsverhältnisse}

Constantins Abhängigkeiten und Ängste spiegeln wider, wie es zahlreichen gewaltbetroffenen Kindern und Jugendlichen oft nach einem nicht aufgearbeiteten Abbruch missbräuchlicher Beziehungsverhältnisse geht. Die Bindung an Täter*innen wirkt in den Betroffenen zunächst fort (Alaggia \& Mishna, 2014). In 
Constantins Fall kommt hinzu, dass der Täter einen Männerbund (,,echte Männersachen") inszenierte, der an gemeinsame technische Interessen anknüpft. In solchen Fällen kann Technik als Mittel der Aufwertung des Männlichkeitsstatus, der Abgrenzung gegen vermeintliche Nichtmännlichkeit und einer intensiveren Bindung an Täter*innen dienen (Vobbe \& Kärgel, 2020). Im Falle Constantins ist ferner denkbar, dass die Einwahl in das Tor-Netzwerk als illegale Handlung ritualisiert wurde, zum Beispiel indem P. kriminelle Angebote oder Gewaltpornografie suchte und aufrief. Entsprechende Verhaltensweisen erhöhen den Geheimhaltungsdruck auf betroffene Kinder und Jugendliche, da sie sich in ihrer eigenen Wahrnehmung an verbotenen Handlungen beteiligt haben. Täter*innen fällt es dann leichter, Betroffenen zu vermitteln, sie seien Teil einer eingeschworenen Gemeinschaft und von anderen schützenden Personen isoliert. Zugleich desensibilisieren Gewaltausübende Betroffene und bereiten weitere Grenzverletzungen vor. Männlichkeitsriten und -bünde begünstigen die Abspaltung und Verdrängung von Gewalt, da sie sexualisierte Übergriffe in den Bedeutungskontext von Zusammenhalt, Eingeschworen-Sein und Exklusivität rücken (ebd.).

\begin{abstract}
„Eine gemeinsame Interpretation von Männlichkeit bestand darin, sich für Technik zu interessieren und wahrscheinlich auch darin, gemeinsam Pornos zu gucken. [...] Den ,rückständigen“ und ,biederen ‘ Müttern durfte natürlich nichts erzählt werden - und gegenüber S., die zuvor mit ihren Brüdern [...] noch in einem guten Kontakt stand, fand eine schleichende, wenn auch nicht zu übersehende Entfremdung statt.“
\end{abstract}

Falldokumentation 6.2

Abzuklären ist, ob Constantins Ängste technisch begünstigt werden, indem P. dem Jungen beispielsweise suggeriert, dass er ihn beobachten kann. Wie in Kap. 9 erörtert wird, löst mediatisierte sexualisierte Gewalt alltagsbegleitende Ängste aus. Constantins Angst hat paranoide Züge („Constantin will wissen, ob P. erfährt, was in der Beratung besprochen wird"). Insbesondere Prometheus wirkt in der Wahrnehmung des Jungen sehr machtvoll. Gehören Drohstrategien zum Verhaltensrepertoire von Täter*innen, um eine Aufdeckung der Gewalt zu verhindern (Schröder et al., 2020), kann eine Drohkulisse dadurch verstärkt werden, dass Täter*innen Gewaltbetroffenen technische Kontrollmöglichkeiten vorführen oder sie diese spüren lassen. Einem Jungen die Möglichkeiten einer Drohne mit hochauflösender Kamera darzubieten, würde sich hierfür genauso eigenen wie ihn tatsächlich zu überwachen, etwa mit der Installation von Überwachungssoftware auf dem Smartphone, einer Weiternutzung von Login-Daten für gemeinsam eingerichtete Soziale Medien, Hacking oder die heimliche Installation von Kameras (Hartmann, 2017). Wirkungsvoll ist die Täter*innenstrategie auch dann, wenn für 
Betroffene nicht nachvollziehbar ist, welche Mittel zu ihrer Überwachung tatsächlich eingesetzt werden können. Im Gegenteil dürfte die Mischung aus Suggestion und Unsicherheit sogar noch ängstigender wirken.

\subsubsection{Hinweise auf organisierte und rituelle Gewalt}

Unter organisierter Gewalt verstehen wir Formen (sexualisierter) Ausbeutung durch vernetzte Täter*innenkollektive. Häufig werden in diesem Zusammenhängen Missbrauchsabbildungen zu kommerziellen Zwecken hergestellt und verbreitet sowie sexueller Kindesmissbrauch als „Dienstleitung“ angeboten (Kinderund Zwangsprostitution) (Nick et al., 2019). Das Ziel, anhand sexualisierter Ausbeutung ein gewalttätiges Konsumangebot kommerziell zu vertreiben, begründet in Kombination mit der Vernetzung untereinander die Nähe organisierter Täter*innen-Strukturen zu digitalen Medien. Seit den Anfängen des World Wide Web werden Foto-, Video- und Kommunikationsmedien von Täter*innenNetzwerken pädokriminell ${ }^{2}$ genutzt, um Missbrauchsabbildungen herzustellen, zu verbreiten und sexuellen Kindesmissbrauch zum Konsum anzubieten. Der technische Vorsprung gegenüber Strafverfolgungsbehörden ohne eine Spezialisierung im Bereich Cybercrime und ein hohes Maß an krimineller Organisation erklären die seltenen Ermittlungserfolge gegen entsprechende Kollektive (Igney \& Kreyerhoff, 2018).

Ist organisierte Gewalt (pseudo-)ideologisch eingebettet, sprechen wir von ritueller Gewalt. Ideologie erfüllt den Zweck, Gewalt zu rechtfertigen. Sie erschwert den Ausstieg Betroffener, da Letztere - wie im Zusammenhang anderer Psycho-Gruppen - durch das Wegbrechen des weltanschaulichen Systems einen Sinn- und Bedeutungsverlust erleben. Ideologie ist ebenso Teil der mystischen, teils magischen Selbstinszenierung der (All-)Macht und Kontrolle von Täter*innen über Betroffene (Igney \& Kreyerhoff, 2018; Nick et al., 2019). Im Zusammenhang ritueller Strukturen berichten Betroffene sowie Beratende auch häufig von einer gezielten Spaltung von Persönlichkeitsanteilen durch schwere (sexualisierte) Gewalt und Ängstigung. Bereits bei Kleinkindern provozieren Täter*innengruppen dazu mittels Gewalt Dissoziationen und dissoziative Identitätsstörungen ${ }^{3}$.

\footnotetext{
${ }^{2}$ In Anlehnung an Brachmann (2016) sprechen wir bei (sexualisierten) Verbrechen gegen Kinder von Pädokriminalität.

${ }^{3}$ Von einer dissoziativen Identitätsstörung im pathologischen Sinne sprechen wir, wenn unterschiedliche Persönlichkeitszustände eines Menschen dessen Gefühle, Handlungen und
} 
„Die Stimme und auch die Stimmung wechselten mehrfach während des Gespräches. Am Ende war ich sehr unsicher, ob die Anrufende wirklich 17 Jahre alt ist oder nicht eher doch schon älter. Vielleicht hat mich schlicht der 17-jährige Anteil angerufen.“

Falldokumentation 6.3

Unterschiedliche Persönlichkeitsanteile Betroffener werden bisweilen durch Täter*innen für verschiedene Zecke trainiert und können per Auslösereiz aktiviert werden. Von dieser Art Steuerung spricht man als Mind Control (Nick et al., 2019). Dazu gehört auch, dass Betroffene selbst in kriminelle Handlungen (etwa Drogenhandel oder Handel mit Missbrauchsabbildungen) und die Rolle von Täter*innen gedrängt werden. Die starke Bindung an die Gewaltstrukturen, eine organisierte Geheimhaltung der Gewalt, die Verbindungen von Täter*innen in gesellschaftlich einflussreiche Milieus und ein gesellschaftlicher Unglaube, dass derart extreme Formen von Gewalt - gezieltes Erwirken dissoziativer Persönlichkeitsstörung (bei Kleinkindern), erzwungene Nahtoderfahrung, transgenerationale sexualisierte Folter, systematische Gruppenvergewaltigung, sogenannte Kinderprostitution und kommerzielle Herstellung von Missbrauchsabbildungen - existieren, erschweren ihre Aufdeckung (Nick et al., 2019; Schröder et al., 2020).

Die gegebene Fallstruktur erlaubt die Beratungshypothese organisierter Gewalt, weil Constantin Opfer schwerer mediatisierter sexualisierter Gewalt durch eine Person wurde, die vernetzt mit anderen Personen agierte. Es wurden Abbildungen seines sexuellen Missbrauchs hergestellt und in Anbetracht der weiteren aufgefundenen Missbrauchsabbildungen wahrscheinlich verkauft, getauscht und/oder verbreitet. Assoziationen ritueller Gewalt ergeben sich durch Bezüge zu Symbolen und Figuren (Prometheus, Ikarus, Büchse der Pandora), denen in der antiken, griechischen Mythologie eine besonders machtvolle zivilisatorische Bedeutung zukommt (vgl. Léfèvre, 2003). Constantins Äußerung ,einen sicheren Freund erkennt man in unsicherer Sache“ ist einem Zitat Ciceros entlehnt.

D: „Ja, nee, ich meine jetzt nur, wenn es was tatsächlich unter Umständen in irgendeiner Form Rituelles ist, wäre es ja toll, wenn es aufgedeckt würde mal.“

A: „Was ist denn der Prometheus?“ “ [...]

B: „Fast unsterblich.“

C: „Fast, ne, ja.“

Wahrnehmung vollständig bestimmen. Die Teilidentitäten verfügen über eigene, völlig unterschiedliche charakterliche Eigenschaften und Fähigkeiten. Ihre Ursache sind zumeist traumatische Erlebnisse, deren Folge die Abspaltung ist (Sar, 2016). 

A: „Hierarchische Stellung dann, ne.“
C: „Und gottgleich, ne.“
A: „Herausgehobene Stellung.“
C: „Wohltäter der Menschheit, Gegenspieler des tyrannischen Zeus. Also, da hat er sich wirklich riesengroß inszeniert, ne, und hat es geschafft, dass der Constantin ihn offenbar auch in dieser Rolle so sieht und akzeptiert, ne, den Prometheus."

Traumafachberaterin, spezialisierte Fachberatung rituelle Gewalt, in der Diskussion mit Spezialermittler, Rechtspsychologe und Kinder- und Jugendpsychotherapeutin, FGI

Wäre Constantin von organisierter oder ritueller Gewalt betroffen, sind das Ausmaß seiner Belastungen, Ängste und möglichen Traumatisierung, die Bindung an die Gewaltstrukturen, die Schwierigkeit einer Aufarbeitung der Gewalt sowie mögliche Täter*innenintrojekte ${ }^{4}$ als fachlich besonders herausforderungsvoll einzustufen. Im Falle ritueller Gewalt muss prinzipiell damit gerechnet werden, dass Gewaltbetroffene auch während der Beratung, Aufarbeitung und Therapie noch im Kontakt zu Täter*innenstrukturen stehen (Igney \& Kreyerhoff, 2018; Nick et al., 2019). Dazu trägt bei, dass Persönlichkeitsanteile Betroffener täter*innenloyal konditioniert werden können und in Konkurrenz zu anderen Anteilen einen Ausstieg sabotieren. So erklärt sich, weshalb Betroffene von Täter*innen gezwungen werden können, aus Beratungs- oder Psychotherapiesitzungen $\mathrm{zu}$ berichten und für Ausstiegsversuche bestraft werden (Nick et al., 2019). Ziel dieser Täter*innenstrategie ist vor allem zu erfahren, ob der Ausstieg einer betroffenen Person Täter*innenstrukturen gefährdet.

Offen bleibt, ob der von Constantin gezeigte Blogeintrag im Gaming-Forum (,Ikarus_1.0“) ein Versuch des Jungen ist, unsagbare Gefühle und personifizierte alltagsbegleitende Ängste (,,eisige Tentakel“, „Platzangst“, ,Du bist nicht einer. Du bist viele.") in mediatisierten Kontexten auszudrücken, ${ }^{5}$ oder ob es sich um einen Eintrag handelt, der von den Täter*innen stammt und als Warnung oder als

\footnotetext{
4 Täter*innenintrojekte sind psychische Leitbilder und Verhaltensweisen, die Betroffene durch die Gewalt verinnerlicht haben. Das können Selbstabwertungen, Abwertungen anderer Menschen, selbstverletzendes Verhalten oder andere Verhaltensweisen sein, die eine Fortsetzung der Bindung zur gewaltausübenden Person darstellen. Vereinfacht ausgedrückt handelt sich um Reinszenierungen der Bindungskommunikation mit dem*der Gewaltausübenden. Die darin ausgedrückte Identifikation mit übergriffigen Verhaltensweisen ist psychodynamisch ein Schutz vor der eigenen Hilflosigkeit und Ohnmachtsabwehr (Peichl, 2015).

${ }^{5}$ Beispiele solcher Einträge und Memes finden sich unter anderem in Müller und Lüttichau (2019a, b). Die Beispiele dort sind ungeachtet der Kontroversen um den Herausgeber (von Lüttichau) des Buchs authentisch.
} 
Auslösereiz für täter*innenloyale Persönlichkeitsanteile Constantins zu verstehen ist.

\subsection{Handlungsempfehlungen}

Für die nachfolgenden Handlungsempfehlungen gehen wir weiter davon aus, dass Constantin von ritueller Gewalt betroffen ist. Diese Beratungshypothese soll uns vor einer voreiligen Reduktion der möglichen Fallkomplexität und einer Bagatellisierung der Gewaltwiderfahrnisse Constantins schützen.

„Ist es ritueller Kontext, bedarf es einer anderen Art des Arbeitens. Ist er noch gefährdet, gefährdet er andere? [...] Wenn ich das weiß, dann würde ich vielleicht anders arbeiten.“

Traumafachberaterin, spezialisierte Fachberatung rituelle Gewalt, FGI

Unsere Hypothese ist nicht als Gewissheit über den Gewaltkontext misszuverstehen und muss im Rahmen der konkreten Fallarbeit weiterentwickelt werden. Jedoch erscheint es uns leichter, die Maßstäbe fachlicher Komplexität im Umgang mit ritueller Gewalt schrittweise abzusenken, sollten sich Teilaspekte dieses Worst-case-Szenarios nicht bestätigen, als umgekehrt zu riskieren, dass scheinbare Teilerfolge durch übersehene Fallkomponenten unterwandert werden. Dazu ziehen wir in Erwägung, dass

- der Besitz der Gewaltvideos auf mediatisierte Kontroll- und Verarbeitungsversuche Constantins hinweisen könnte, es sich aber genauso um Formen der Selbstbestrafung, eigene Täter*innenintrojekte oder um ein Drohmittel von Täter*innen handeln könnte,

- Constantin nach wie vor Abhängigkeiten zu dem/der/den Täter*innen erlebt, welche sich im Rückgriff auf die Symbolik und Constantins Bindung an eine (Schein-) Ideologie zeigen,

- Constantin weiterhin durch Täter*innen kontaktiert werden könnte oder zumindest Angst davor hat,

- Constantin eine dissoziative Identitätsstörung mit täter*innenloyalen Anteilen haben kann, die selbst Täter*innen kontaktieren und gegebenenfalls sogar aus Beratungs- und Therapiesitzungen berichten,

- der Blogeintrag ein Versuch Constantins ist, seine personifizierte Angst im Kontext digitaler Medien auszudrücken oder aber der Eintrag vielmehr eine Schnittstelle zu Täter*innenanteilen oder Täter*innen darstellt. 
Die Arbeit mit Constantin setzt eine ganzheitliche Perspektive voraus, die sich der enormen Herausforderungen ritueller Gewalt sowie der Grenzen diesbezüglicher professioneller Möglichkeiten bewusst ist, ohne sich der Hoffnungslosigkeit hinund Constantin aufzugeben. Wir blenden zwecks Reduktion weiterer Komplexität Möglichkeiten der Strafverfolgung und rechtliche Aspekte aus, weil diese a) auf Basis der gegenwärtigen Faktenlage nicht ohne vorherige Aufarbeitung mit dem Adressaten denkbar sind und b) ihn sogar gefährden könnten, da organisierte und rituelle Strukturen sich selbst schützen. Wir verbleiben stattdessen radikal beim Betroffenen. Rode (2016) bezeichnet die Arbeit mit Betroffenen ritueller Gewalt daher als Gratwanderung, weil der Spagat zwischen Unterstützung der Person und professioneller Abgrenzung gegen die Logik der Gewaltsysteme besonders spannungsgeladen ist.

Eine hierfür notwendige Haltung finden wir in Konzepten der systemischen Traumapädagogik. In Anlehnung an die Salutogenese betrachtet die systemische Traumapädagogik Gesundheit als kontinuierlichen Herstellungsprozess zwischen lebenden Systemen (Individuen, aber auch sozialen Systemen) und ihrer Umwelt, sprich als etwas Dynamisches. Sie greift dazu auf die Metapher eines Flusses zurück. Traumatische Erfahrungen in der Kindheit vergleicht sie mit Strudeln, in die Menschen geraten oder gestoßen werden, die noch nicht richtig schwimmen können. Es bleiben Versuche zurück, sich über Wasser zu halten (Jegodtka \& Luitjens, 2016). Mit der Metapher werden weder wichtige Erkenntnisse der klinischen sowie der Neuropsychologie missachtet noch die Komplexität von Traumata vereinfacht. Vielmehr dient sie dazu, eine Haltung auszurichten, die auch eine komplexe Traumatisierung nicht ausschließlich aus defizitorientierter Perspektive sieht. Sie nimmt mehrdimensionale ,ökologische“ Ressourcen in den Blick, die dem verlorenen Kohärenzgefühl Betroffener entgegenwirken und sie stützen, gute Schwimmer*innen zu werden.

Die nachfolgenden Maßnahmen und Interventionen verstehen wir als Bemühung um „gewaltfreie Räume“, die - wie noch erörtert wird - psychosoziale, mediatisierte, leibliche und institutionelle Aspekte in ihrer Wechselwirkung betrachtet. Wir dürfen uns „gewaltfreie Räume“ als Geflecht mit Ausgängen und Verbindungsgängen vorstellen, mit denen ein Wechsel der Handlungsebene möglich ist. 


\subsubsection{Pädagogische und beraterische Beziehungsarbeit}

Die systemische Traumapädagogik geht insbesondere im Falle von sexualisierter Gewalt davon aus, dass betroffene Kinder und Jugendliche einen Vertrauensverlust und eine Desillusionierung in Beziehungen erleben. Um Adressat*innen dabei zu unterstützen, sich weiterzuentwickeln, müssen sie gemäß traumapädagogischen Prinzipien stabile Beziehungen zu Helfenden aufbauen. Der Leitsatz lautet hierbei: Ein „Ich“ entwickelt sich permanent in der Begegnung mit dem „Du“ (Jegodtka \& Luitjens, 2016). Im Falle Constantins ist bei der Beziehungsarbeit besonders zu berücksichtigen, dass er Abhängigkeits- und Loyalitätskonflikte erlebt und seine Ängste vor P. durch die Möglichkeit digitaler Interaktionen sowie das Trigger-Potenzial digitaler Medien verstärkt bzw. reaktiviert werden. Der Berater und auch die Sozialpädagogin der betreuten Wohngruppe müssen darauf achten, dass sie Constantin ein Verlässlichkeitsangebot ${ }^{6}$ machen, welches nicht in Konkurrenz zu dem Täter*innenkollektiv steht. Andernfalls riskieren sie, den Jungen in ein Entscheidungsdilemma zu führen - entweder für die Helfenden oder die Täter*innen - und einen Kontaktabbruch. Insofern ist die Intervention des Beraters, „was P. außerdem unternommen habe, um Constantin zu täuschen“, riskant. Zwar kann hierin der Versuch einer Normveränderung erkannt werden. Diese erfolgt jedoch offenbar zu einem zu frühen Zeitpunkt in der Verarbeitung Constantins. Sie wäre außerdem eher als beraterisches Resonanzangebot statt als unmittelbares Bedeutungs-Reframing des Verhaltens P.s zu gestalten: „Ich denke gerade, manchmal tun Erwachsene aber auch nur so, als würden sie sich besonders viel Mühe um ein Kind geben. Für das Kind ist es dann nämlich viel schwieriger Nein zu sagen, wenn der Erwachsene etwas von ihm verlangt, was es nicht will. Trotzdem fühlt sich das Kind hinterher schlecht. Es will den Erwachsenen ja nicht enttäuschen. Kennst du sowas auch?“”

Im Falle Constantins gilt es zudem zu berücksichtigen, dass täter*innenloyale Anteile eine Aufdeckung des Täter*innen-Netzwerks aktiv sabotieren. Unter Expert*innen ist daher umstritten, wie weit die Zusicherung einer Adressat*innenorientierung gehen kann, ohne Schweigegebote und damit das Normsystem der Täter*innen zu stützen. Gleichzeitig ist damit zu rechnen, dass Ratsuchende die Vertrauenswürdigkeit Helfender testen. Insofern entscheidet das konkrete Verhalten und nicht Versprechungen über den Erfolg des Beziehungsaufbaus. Constantin sollte zurückgemeldet werden, dass er zu keinem Zeitpunkt

\footnotetext{
${ }^{6}$ Da sexualisierte Gewalt von Kindern und Jugendlichen zumeist auch als Missbrauch von Vertrauen erlebt wird, sprechen wir uns dafür aus, pädagogische Beziehungen nicht in erster Linie als Vertrauensbeziehungen, sondern als verlässliche Angebote zu verstehen. Sofern dies zu einem Aufbau von Vertrauen führt, schadet dies den Adressat*innen nicht.
} 
gezwungen wird, etwas zu erzählen und er sämtliche Gesprächsinhalte so wählen kann, dass er sich nicht belastet. Der Leitsatz lautet: „Ich arbeite mit dir an deinen Themen. Du musst mir nichts erzählen, was du nicht willst, wenn es dich oder andere belastet oder es dich ängstigt“. Der Berater sollte Constantin außerdem zusichern, dass weder P. noch andere*r Täter*innen und Dritte erfahren werden, was in der Beratung besprochen wird.

Da Constantin ein Jugendlicher ist und damit unter besonderem Schutz steht, muss ihm erklärt werden, unter welchen Umständen der Berater oder die Sozialpädagogin die Notwendigkeit sehen, sich aufgrund des Schutzauftrags bei Kindeswohlgefährdung nach $\S 8$ a SGBVIII oder eines rechtfertigenden Notstands nach $\S 34 \mathrm{StGB}$ mit anderen Personen zu beraten. Wenngleich vor diesem Hintergrund zu vermuten ist, dass Constantin bisweilen abwägt, was er erzählen wird, ist die Offenlegung der professionsethischen Verpflichtung der Helfenden ein Ausdruck von Authentizität und Verlässlichkeit. Täter*innenloyale Anteile Constantins müssen dafür nicht moralisch abgelehnt werden (Becker et al. 2019a, b). Im Gegenteil umfasst sie eine ganzheitliche Annahme des Jungen.

\subsubsection{Traumapädagogische Diagnostik}

Im Falle Constantins bietet sich eine traumapädagogische Gestaltungsdiagnostik an. Im Sinne unseres systemischen Traumaverständnisses umfasst diese sowohl eine klassifikatorisch-psychiatrische, eine biografische sowie eine Lebensweltdiagnostik. Zusammengenommen führt sie in ein Koordinatensystem biopsychosozialer Diagnostik, die Umgebungsfaktoren, Stressoren und Belastungen, individuell personale Faktoren sowie Stärken und Ressourcen partizipatorisch abbildet. Bei aller gebotenen Kritik der Traumapädagogik an der Psychopathologie sollte die klassifikatorisch-psychiatrische Diagnostik als eine Lesart der Belastungen des Adressaten wahrgenommen werden (Gahleitner, 2017). Aufgrund der rituellen Gewalterfahrungen richtet sich unser Interesse besonders darauf, ob eine dissoziative Persönlichkeitsstörung (DIS) vorliegen könnte.

\subsubsection{Gewaltfreie Lebensräume schaffen}

Die Schaffung eines gewaltfreien Lebensraums erscheint in Fällen mediatisierter sexualisierter Gewalt grundsätzlich schwierig, da die erneute Konfrontation mit digitalen Gewaltzeugnissen (z. B. gespeicherte Chatverläufe, archivierte EMails, Missbrauchsabbildungen) oder eine Kontaktaufnahme durch Täter*innen 
vor dem Hintergrund permanenter Erreichbarkeit mittels digitaler Medien nicht ausgeschlossen werden können. In Fällen organisierter und ritueller Gewalt ist das Risiko einer Kontaktaufnahme und Einschüchterung Betroffener durch die Täter*innen als besonders hoch einzuschätzen. In Constantins Fall kommt erschwerend hinzu, dass er selbst Gewaltabbildungen besitzt. Ferner sollte in Erwägung gezogen werden, dass täter*innenloyale Anteile mittels digitaler Medien Kontakt zu den Täter*innen aufnehmen. Die Vorstellung eines einheitlichen Lebensraums, der gegen jedes Eindringen von Gewalt geschützt ist, muss daher der Vorstellung einer Pluralität von Räumen weichen, die als unterschiedlich gewaltbehaftet oder gewaltfrei erlebt werden.

Ein wichtiger Ratgeber in diesem Raumgeflecht ist Constantin selbst. Die Forschung und Praxiserfahrungen zu ritueller Gewalt unterstreichen, dass das Sicherheitsbedürfnis Betroffener umgekehrt proportional zu ihrem permanenten Bedrohungserleben sehr groß ist (Igney \& Kreyerhoff, 2018). Constantin kann also angeregt werden, über die Fragen, wo und wie er sich sicher(er) oder weniger sicher fühlt, mitzuentscheiden. Im systemischen Sinne denken wir hierzu sein subjektives Erleben, seine Beziehungsnetzwerke, digitale Medien und die strukturell-institutionelle Ebene sozialräumlich zusammen.

Ob beispielsweise die Unterbringung in einer betreuten Wohngruppe und die Haft P.s dazu beitragen, dass Constantin örtlichen Schutz erlebt, kann gemeinsam mit ihm reflektiert werden. So dürfte es den Gewaltausübenden schwerer fallen, Constantin jenseits digitaler Medien in der Einrichtung aufzusuchen. Freilich wird das Sicherheitsempfinden des Jungen stark davon abhängen, dass die Institution a) nicht als Täter*inneneinrichtung wahrgenommen wird, b) über Grundkenntnisse zu organisierter oder ritueller Gewalt und c) über ein traumapädagogisches Konzept verfügt. Andernfalls ist der Wechsel in eine andere Einrichtung zu erwägen. Auch die Beziehungen zur Sozialpädagogin und zum Berater können prinzipiell von Constantin als gewaltfreie Sozialräume wahrgenommen werden. Die Sozialpädagogin vermittelt Constantin beispielsweise an eine Beratungsstelle, statt ihn für den Besitz von Gewaltvideos zu sanktionieren. Dies spricht für ihre Gewaltsensibilität. Auch in ihrem und im Falle des Beraters entscheidet zudem der gelingende Aufbau weiter oben genannter Beziehungsarbeit über Constantins Schutzerleben.

Inwieweit digitale Medien von Constantin geschützt gewaltfrei genutzt werden können, ist schwerlich vorherzusagen. In den Focus Group Interviews wird hierüber ohne finalen Konsens diskutiert.

\footnotetext{
„Also, wenn das eine permanente Bedrohung für den Constantin oder für wen auch immer, ne, der retraumatisiert wurde im digitalen Raum, wäre. [...] Ich finde, darüber müsste dann auch eben die professionelle Diskussion sich drehen, um die Frage,
} 
inwieweit muss man sich dem aussetzen. [...] Das Ideale wäre ja, wenn Menschen, die Opfer sexualisierter Gewalt im digitalen Raum wurden, nicht komplett auf Medien verzichten, auf digitale Medien verzichten müssen.“

Rechtspsychologe, FGI

„Das ist aber nochmal ein Unterschied, ob ich das mit einem besprechen kann, der Aussteiger sein will, aus dieser, aus so einer Szene und da vielleicht auch mal eine gewisse Zeit auf alles mit Digitalem verzichten muss, um so auch nicht erreichbar zu sein.“

Traumafachberaterin, spezialisierte Fachberatung rituelle Gewalt, FGI

„Ich weiß nicht, wie realistisch das ist, so komplett ohne irgendwie digitale Medien [...]. Wenn man sich jetzt gesamtgesellschaftlich die Entwicklungen anguckt, ob das, ja, wie vereinbar das dann ist oder was das für Konsequenzen hat."

Präventionsfachkraft, FGI

\subsubsection{Abwägungen zur Mediennutzung}

Fragen nach einem Umgang mit Constantins Mediennutzung behandeln wir entlang zweier Schwerpunkte, a) des Besitzes der Missbrauchsabbildungen sowie b) Constantins Verweis auf den Blogeintrag. Der Besitz der Missbrauchsabbildungen ist fachlich problematisch zu bewerten. Die Videos widersprechen der Vorstellung gewaltfreier Räume, reaktivieren traumatische Belastungen, normalisieren Gewalt und womöglich auch Täter*innenintrojekte. Ungeachtet dessen besteht das Risiko, dass Constantin sich strafbar macht, sollte das Material juristisch als sogenannter kinderpornografischer Inhalt im Sinne von § 184b StGB bewertet werden. Mit der Möglichkeit, dass es sich um Material handelt, dass ihm zur Bedrohung zugeschickt wurde, beschäftigen wir uns unten.

Demgegenüber darf nicht vergessen werden, dass die Beschäftigung mit oder die Suche nach dem Material Constantin womöglich im Sinne maladaptiven Self-Triggering vorübergehend Kontrolle und Spannungsabbau verschafft. Die beraterische Auseinandersetzung mit dem Material sollte, wie die Arbeit mit von ritueller Gewalt Betroffenen prinzipiell, nicht von moralischen Zuschreibungen an das Medienhandeln Gewaltbetroffener geleitet sein, sondern von einem gemeinsamen Verstehensprozess (Rode, 2016). Handlungsleitend sind folgende - nicht direkt an Constantin gerichtete - Fragen:

- Weshalb besitzt/sucht/nutzt Constantin das Material?

- Was erlebt er vor, während und nach einer Suche/Nutzung? 
- Welche Zusammenhänge gibt es zwischen den männerbündischen Ritualen P.s und dem Besitz/der Nutzung des Materials?

- Inwiefern ent- und belastet ihn die Beschäftigung mit den Videos?

- In welchen anderen Situationen erlebt Constantin vergleichbare Be- oder Entlastung?

- Welche Handlungsalternativen ergeben sich hieraus?

- Welche Zusammenhänge bestehen zwischen Constantins Medienhandeln und Ängsten?

Im Sinne Weiß' (2017) werden Selbstregulation, Ermächtigung im Sinne eines Selbstverständnisses des eigenen Körpers als Frühwarnsystem, ein Erkennen von Triggern und Selbstakzeptanz als Schutzfaktoren gefördert. Wir erneuern in dem Zusammenhang den Grundsatz der Rode'schen Gratwanderung unserer Arbeit mit Betroffenen von ritueller Gewalt, schließlich bleibt das Material problematisch. Ermächtigung bedeutet daher auch, den Jungen über das in den Videos gezeigte Unrecht gegen die Integrität von Kindern, seinen eigenen Anspruch auf Hilfen und Schutz vor sexuellem Missbrauch oder Folterung und die mögliche Strafbarkeit der Aufnahmen, ihrer Herstellung und Verbreitung aufzuklären. Die demonstrierte Haltung korrigiert erlebte Grenzverletzungen, ohne Constantin als Person abzuwerten. Sie ist die Grundlage, um Handlungsalternativen gemeinschaftlich zu erarbeiten und zu erproben.

Diffus ist weiterhin der Blogeintrag. Über seine Bedeutung ist mit Constantin entlang der skizzierten Grundsätze zu verhandeln. Die verwendete Symbolik ist zu verworren, als dass ein fundiertes Urteil über dessen Sinngehalt für Constantin gefällt werden kann.

„Ich finde, es ist eine gute Grundlage, um zu arbeiten. Das ist Ausdruck eines Gefühls, klar, es ist dark, aber es ist Kommunikation, es ist ein Angebot, damit kann man arbeiten. Und für mich wäre es das Nächstliegende, den Constantin zu fragen, welche Bedeutung hat das für dich, ne, ist jetzt, kannst du es mir erklären, was ist das oder liege ich richtig, wenn ich es so und so deute."

Rechtspsychologe, FGI

Mit der Möglichkeit der Kontaktaufnahme durch/mit Täter*innen beschäftigen wir uns noch. Zuvor stellen wir die Beratungshypothese auf, dass sich Constantin insoweit mit dem Eintrag identifiziert, als dort für ihn (noch) unaussprechliche Ängste zum Ausdruck gebracht werden. Die Anspielungen auf ein übermächtiges Kollektiv („Du bist nicht einer. Du bist viele. Legion“, „Tentakel“) und die Assoziationen technologischer Singularität („Blackbox“, „KI“, „Pupillen im 
Interface“, „Puppet Master“) hinterlassen den Eindruck, als personifizierten digitale Medien ein bedrohliches Kollektiv. Die Hypothese stützt sich darauf, dass a) P. Constantin vor allem mediatisierte Angebote machte, b) Täter*innen ritueller Gewalt Betroffenen suggerieren, sie seien dazu in der Lage, sie zu jeder Zeit und an jedem Ort zu kontrollieren und c) digitale Medien sich zwecks Herstellung dieses Glaubens eignen, die behauptete Allmacht von Täter*innen durch kultisch-magische Ideologiegerüste zu überhöhen (Becker et al. 2019a). Da die Möglichkeit besteht, dass Täter*innen Constantin kontaktieren, führt die Beschäftigung mit dem Blogeintrag und Constantins Ängsten im Kontext digitaler Medien erneut zu der vergegenwärtigten Gratwanderung. Während der Sinn des Blogeintrags mit Constantin zu erarbeiten ist und darin verborgene Hinweise auf Kontrollmöglichkeiten der Täter*innen ernst zu nehmen sind, sollte ihm ein Resonanzangebot gemacht werden, das den Glauben an eine Allmacht der Täter*innen relativiert. Eine spezialisierte Beraterin zu ritueller Gewalt, die im HUMAN-Projekt befragt wurde, formuliert dies so: „Manche Erwachsenen kennen miese Tricks, um Kinder oder Jugendliche abzuhören oder zu kontrollieren. Man fragt sich dann, woher die dieses und jenes wissen. Das wirkt sehr mächtig und beängstigend. Genau das wollen die auch. Aber es sind eben Tricks. Menschen, die alles abhören können oder wissen, die gibt es nämlich nicht". Entsprechend dem Ansatz des gewaltfreien Ortes kann mit Constantin unterschieden werden, mit welcher Form digitaler Mediennutzung er sich geschützt fühlt, welche Begleitung er in diesem Zusammenhang wünscht und wann er Unsicherheit erlebt. Dazu kann auch gehören, sein Smartphone und andere mobile Endgeräte in Abstimmung mit ihm durch einen IT-Dienstleister auf Überwachungs-Software durchsuchen zu lassen.

Selbst erlebte dysfunktionale Verhaltensweisen, zum Beispiel die Suche nach Triggern oder Gewaltabbildungen, und die Angst, Kontakt zu Täter*innen aufzunehmen, könnten ergänzend zur Traumapädagogik durch Dialektisch Behaviorale Therapie $(\mathrm{DBT})^{7}$ begleitet werden. Die DBT erarbeitet mit Adressat*innen Fähigkeiten, um dysfunktionales Verhalten zu ändern, Kontingenz herzustellen, verzerrtes Denken umzustrukturieren und gleichwohl negativ erlebte Affekte zu tolerieren. Das Wort ,dialektisch“ verweist auf zwei scheinbare Gegensätze des Konzepts, nämlich den verhaltenstherapeutischen Ansatz, Fähigkeiten zur Veränderung einer Situation erlernen zu können, zugleich aber zu akzeptieren, was nicht veränderbar ist. Die vermittelten Fähigkeiten folgen der Zielsetzung einer Neuausrichtung der Aufmerksamkeit, des Akzeptierens von Erfahrungen

\footnotetext{
${ }^{7}$ Zur Einführung in die Behandlung von Traumata mittels DBT empfehlen wir die Lektüre des Beitrags von Sweezy (2011).
} 
sowie einer Emotionsregulation auf der Basis nicht wertenden Beobachtens. Einzeltherapeutische Angebote werden durch Tagebuchführung unterstützt. Problematisches Verhalten soll auf diese Weise einschließlich damit verbundener Konsequenzen (Verletzungen, Scham, Ekel, Dissoziation) für Adressat*innen nachvollziehbar und beeinflussbar werden (Sweezy, 2011). Der Ansatz ist klinisch erforscht und eignet sich zur Neuausrichtung maladaptiven Verhaltens (Swerdlow et al., 2020). Seine weltanschauliche Orientierung am Buddhismus und die Zielsetzung, es den Adressat*innen zu ermöglichen, sich selbst zu verstehen und selbstbestimmt zu handeln, passen zu traumapädagogischen Zugängen. Constantin könnte durch eine entsprechende Unterstützung erlernen, welche Formen der Mediennutzung dazu führen, dass er digitale Medien als einen sicheren sowie gewaltfreien Raum wahrnimmt.

Das Risiko, durch Täter*innen kontaktiert zu werden, ist hiermit nicht ausgeräumt. Der Blogeintrag und die gewalthaltigen Videoaufnahmen auf dem Smartphone des Jungen müssen weiterhin als entsprechende Versuche in Erwägung gezogen werden. Dabei kann es sich um Trigger handeln, die der Mind Control des Jungen dienen. Wohngruppen für Betroffene von ritueller Gewalt machen eine Abgabe von Endgeräten (z. B. Smartphone, Tablet) bisweilen zur Zugangsvoraussetzung, die dem Schutz der Betroffenen und der Mitarbeitenden dient. Es handelt sich um eine radikale Maßnahme, die angesichts der Geschlossenheit der Gewaltstrukturen und der Schwierigkeit, diese zu verlassen, nicht mit medienskeptischen Verboten in anderen Fällen mediatisierter sexualisierter Gewalt (s. Kap. 2 und 5) gleichzusetzen ist. Die Verhandlung einer Medienabstinenz, wie sie in den Focus Group Interviews angesprochen wurde, darf eine Option im Umgang mit Constantin sein, wenn dies dem Sicherheitserleben und der Gewaltfreiheit des Adressaten dient. Wir würden uns trotzdem auch in diesem Fall für Selbstbestimmung statt für Verbote aussprechen.

\subsubsection{Teilearbeit}

Die Bezeichnung „Teilearbeit“ wird sehr heterogen verwendet. Selbst in traumazentrierten Kontexten reichen die Ansätze von der Arbeit mit nicht pathologischen Persönlichkeitsanteilen bis zur Therapie dissoziativer Identitätsstörungen. Die Methoden sind sehr komplex und erfordern zumeist eine mehrjährige Ausbildung. Der folgende Abschnitt soll daher dazu anregen, Teilearbeit als eine Haltung der Traumapädagogik zu begreifen.

Die systemische Traumapädagogik geht davon aus, dass alle Menschen verschiedene Teilpersönlichkeiten haben, deren Grad an Dissoziation jedoch variiert 
(Jegodtka \& Luitjens, 2016). Anspruchsvoll in der Arbeit mit Betroffenen ist, dass täter*innenloyale Teilpersönlichkeiten oder Introjekte Beratungserfolge erschweren und teils so programmiert sind, dass sie einen Ausstieg aus den Gewaltstrukturen zu verhindern versuchen. Die systemische Traumapädagogik geht trotzdem davon aus, dass auch oder gerade programmierte Anteile oder Teilpersönlichkeiten als Antwort auf die Lebensanforderungen ihrer Entstehung bzw. Abspaltung zu begreifen sind. So nahe sie dem Gewaltsystem zu stehen scheinen, haben sie mittels Anpassung und Täter*innenidentifikation zum Überleben der Betroffenen beigetragen (Peichl, 2015; Becker et al. 2019b). Teilearbeit zielt in der systemischen Traumapädagogik darauf ab, Allianzen zwischen Helfenden und verschiedenen Anteilen/Teilpersönlichkeiten zu unterstützen. Dazu ist ähnlich der Täter*innenarbeit eine Annahme auch täter*innenloyaler Teilpersönlichkeiten vonnöten. Sie ist nicht gleichzusetzen mit einer stillschweigenden Akzeptanz schädigenden Verhaltens. Allerdings hebt die Haltung die gute Absicht der Anteile/Teilpersönlichkeiten hervor und hält so die Beziehung zu ihnen aufrecht (Peichl, 2015). Eine Integration von täter*innenloyalen Anteilen bis zu ihrer Allianz mit oder Unterordnung unter andere Teilpersönlichkeiten, die sich einen Ausstieg aus dem Gewaltsystem wünschen, ist ein langer Weg, der meist intensiv therapeutisch begleitet wird. Im Zuge beraterischer oder pädagogischer Hilfen entscheidet vielmehr die Haltung der Teilearbeit darüber, als Helfende*r Rückfälle, Grenzüberschreitungen, Selbstverletzung und -gefährdungen Betroffener nicht als persönliches Scheitern zu verstehen oder als Anlass zu betrachten, Adressat*innen aufzugeben. Die Haltung hilft ebenso zu verstehen, weshalb eine Selbstermächtigung Gewaltbetroffener in mediatisierten Kontexten besonders schwierig ist, und kann deshalb ein Grund zur Vereinbarung medienabstinenter Phasen sein.

\subsubsection{Psychohygiene für Helfende}

Eigene Ängste äußerten Expert*innen ausschließlich in den Focus Group Interviews zum Fall Constantins.

„Würde ich auch Angst haben, weil dann würde ich ihn als extremst beängstigend, also mich beängstigend erleben und extremst machtvoll und auch Gewalt, hohes Gewaltpotenzial. [...] Also, ich würde denn sofort die Assoziation, er identifiziert sich mit bestimmten Foltermethoden, und ich wäre nicht mehr geschützt vor ihm und entweder/ dann müsste ich mich schützen. [...] Ich würde mir erst mal eine Supervision holen." 
Kinder- und Jugendpsychotherapeutin, spezialisierte Fachberatung, FGI

Wir betrachten dies als Charakteristikum mediatisierter organisierter und ritueller Gewalt. Laut der Studie von Nick et al. (2019) berichtet zwar lediglich der kleinere Teil Helfender in Kontexten organisierter und ritueller Strukturen, bedroht worden $\mathrm{zu}$ sein $(21,3 \%)$. Die Autor*innen sehen aber einen signifikanten Zusammenhang zwischen den Gewaltstrukturen und einer sekundären Traumatisierung ${ }^{8}$ Helfender. Die Möglichkeit, dass Constantin durch die Schnittstelle digitaler Medien weiterhin in einem Kontakt mit den Gewaltstrukturen steht, führt dazu, dass die Diskutierenden eine Gefährdung als räumlich sehr nah erleben. Freilich besteht diese Möglichkeit. Trotzdem darf die Angst - insbesondere im Setting der Focus Group Interviews - als Hinweis auf Übertragungen verstanden werden. Die Arbeit im Feld setzt deshalb ein besonderes Maß an Reflexion der Dynamiken sekundärer Traumatisierung voraus. Zum Schutz vor sekundärer Traumatisierung und der Psychohygiene Helfender halten Jegodtka und Luitjens (2016) eine traumasensible Organisationskultur und Selbstsorge für notwendig. Maßnahmen einer traumasensiblen Organisationskultur äußern sich in der Unterstützung von Teamzusammenhalt, gemeinsamer Werteentwicklung, der Förderung von Selbstwirksamkeit im Gegensatz zu Entmutigung und einer Bereitstellung angemessener Ressourcen inklusive der Vergütung der Mitarbeitenden. Der Selbstsorge werden Zeit für Freude und Genuss, Methoden zur Distanzierung, Verringerung von Traumaexposition in der Freizeit (z. B. belastende Videos und Literatur ansehen) und zusammenfassend eine Ressourcenorientierung zugerechnet. Im Umgang mit Constantin heißt das, „trotzdem ,ja“ zum Leben sagen“ und ,alles hat seine Zeit - nicht alles geht zu jeder Zeit“ (ebd., 96 f.). Es nützt nichts, dauerhaft die traumatischen Erfahrungen des Adressaten zu fokussieren. Dies wird dem Jungen nicht gerecht und reduziert auch Helfende auf die Rolle von Trauma-Arbeitenden. Den Jungen „,so sein“ zu lassen - also nicht ausschließlich mit der Trauma-Brille zu sehen - ist dagegen auch eine Ressource. Seine Entlastung trägt ebenfalls zu Erfahrungen bei, die entspannend sind und darüber hinaus soziale Beziehungen und gewaltfreie Räume wachsen lassen.

\section{Literatur}

Alaggia, R., \& Mishna, F. (2014). Self psychology and male child sexual abuse: Healing relational betrayal. Clinical Social Work Journal, 42, 41-48. ${ }^{8}$ Unter sekundärer Traumatisierung verstehen wir die Übertragung von Belastungssympto-
men auf Professionelle, die regelmäßig in Trauma- und Gewaltkontexten arbeiten. 
Becker, T., Bialek, J., Mehmel, T., Vogler, A., \& Wichmann, R. (2019a). Traumapädagogik: Das Instrument der Hilfe-/Teilhabeplanung unter Berücksichtigung traumatischer/dissoziativer Prozesse. In M. Huber, E. Kernen, T. Becker, \& G. Plata (Hrsg.), Aus vielen Ichs ein Selbst?: Trauma, Dissoziation und Identität: Tagungsband zur DGTD-Tagung im September 2018 in Mainz. Junfermann Verlag.

Becker, T., Schauer-Kelpin, C., \& Ciecior, A. (2019b). Rituelle und organisierte Gewalt. In M. Huber, E. Kernen, T. Becker, \& G. Plata (Hrsg.), Aus vielen Ichs ein Selbst?: Trauma, Dissoziation und Identität: Tagungsband zur DGTD-Tagung im September 2018 in Mainz. (S. 149-172). Junfermann Verlag.

Bellet, B. W., Jones, P. J., \& McNally, R. J. (2020). Self-triggering? An exploration of individuals who seek reminders of trauma. Clinical Psychological Science, 8(4), 739-755. https://doi.org/10.1177/2167702620917459.

Brachmann, J. (2016). Tatort Odenwaldschule - Ein Werkstattbericht über die Schwierigkeiten der Aufarbeitung von Vorkommnissen pädokrimineller Gewalt in Institutionen. Zeitschrift für Pädagogik, 62(5), 638-655.

Fliß, C., Igney, C., \& von Bracken, R. (2016). Zur Definition Rituelle Gewalt. In C. Fliß \& C. Igney (Hrsg.), Handbuch Rituelle Gewalt: Erkennen - Hilfe für Betroffene - interdisziplinäre Kooperation (3. Aufl., S. 11-18). Pabst Science Publishers.

Fliß, C., Prins, R., \& Schramm, S. (2018). Befreiung des Selbst: Therapiekonzepte zum Ausstieg aus organisierter Ritueller Gewalt. Asanger Verlag.

Gahleitner, S.-B. (2017). Psychosoziale Diagnostik in der Traumapädagogik: Plädoyer für ein qualifizierteres, Diagnostisches Fallverstehen‘. In M. Jäckle, B. Wuttig, \& C. Fuchs (Hrsg.), Handbuch Trauma. Pädagogik. Schule (S. 461-478). transcript.

Hanfland, N. (2011). Defizitäre Bindung als begünstigender Faktor für Sexualdelinquenz bei männlichen Minderjährigen. Kindesmisshandlung und -vernachlässigung, 14(1), 62-79.

Hartmann, A (2017). Fachberatungsstellen und die Digitalisierung geschlechtsspezifischer Gewalt. bff.

Igney, C. (2012). Rituelle Gewalt - im Spannungsfeld von Parallelwelten, gesellschaftlicher (Ab-)Spaltung und psychosozialem Arbeitsalltag. Zeitschrift für Psychotraumatologie, Psychotherapiewissenschaft, Psychologische Medizin, 4, 11-26.

Igney, C. (2019). Sie könnten es sehen. Es ist mitten in der Gesellschaft. Trauma \& Gewalt, 13(2), 160-168. https://doi.org/10.21706/tg-13-2-160

Igney, C., \& Kreyerhoff, A.-M. (2018). Sexualisierte Gewalt in organisierten und rituellen Gewaltstrukturen: Prävention, Intervention und Hilfe für Betroffene stärken. Bundesministerium für Familie, Senioren, Frauen und Jugend.

Jegodtka, R., \& Luitjens, P. (Hrsg.). (2016). Systemische Traumapädagogik. Vandenhoeck \& Ruprecht.

Léfèvre, E. (2003). Studien zu den Quellen und zum Verständnis des Prometheus Desmotes. Vandenhoeck \& Ruprecht.

Müller, M., \& von Lüttichau, M. (Hrsg). (2019a). Wird keiner helfen? Zeugnisse aus der Rituellen Gewalt. Erster Teil. Verlag Autonomie und Chaos.

Müller, M., \& von Lüttichau, M. (Hrsg.). (2019b). Vati hat mich!. Zeugnisse aus der Rituellen Gewalt. Zweiter Teil. Verlag Autonomie und Chaos.

Nick, S., Schröder, J., Briken, P., \& Richter-Appelt, H. (2019). Organisierte und Rituelle Gewalt in Deutschland. Trauma \& Gewalt, 13(2), 114-127. https://doi.org/10.21706/tg13-2-114. 
Peichl, J. (2015). Innere Kritiker, Verfolger und Zerstörer: Ein Praxishandbuch für die Arbeit mit Täterintrojekten (4. Aufl.). Klett-Cotta.

Rode, T. (2016). Gratwanderungen. Beratungsarbeit mit Betroffenen Ritueller Gewalt. In C. Fliß \& C. Igney (Hrsg.), Handbuch Rituelle Gewalt: Erkennen - Hilfe für Betroffene interdisziplinäre Kooperation (3. Aufl., S. 318-332). Pabst Science Publishers.

Sar, V. (2016). Formation and functions of alter personalities in dissociative identity disorder: A theoretical and clinical elaboration. Journal of Psychology \& Clinical Psychiatry, 6(6). doi: https://doi.org/10.15406/jpcpy.2016.06.00385.

Schröder, J., Behrendt, P., Nick, S., \& Briken, P. (2020). Was erschwert die Aufdeckung organisierter und ritueller Gewaltstrukturen? Psychiatrische Praxis, 47(5), 249-259. https:// doi.org/10.1055/a-1123-3064.

Sweezy, M. (2011). Treating trauma after dialectical behavioral therapy. Journal of Psychotherapy Integration, 21(1), 90-102. https://doi.org/10.1037/a0023011.

Swerdlow, B. A., Pearlstein, J. G., Sandel, D. B., Mauss, I. B., \& Johnson, S. L. (2020). Maladaptive behavior and affect regulation: A functionalist perspective. Emotion, 20(1), 75-79. https://doi.org/10.1037/emo0000660.

Vobbe, F., \& Kärgel, K. (2020). Sexualisierte Gewalt mit digitalem Medieneinsatz gegen Jungen: Geschlechterbezogene Risiken und Herausforderungen für die Prävention. Theorie und Praxis der Sozialen Arbeit, 1, 49-56.

Weiß, W. (2017). Quo vadis Traumapädagogik? Inspirationen, Konzepte, Fragen. In M. Jäckle, B. Wuttig, \& C. Fuchs (Hrsg.), Handbuch Trauma. Pädagogik. Schule (S. 634654). Transcript.

Open Access Dieses Kapitel wird unter der Creative Commons Namensnennung 4.0 International Lizenz (http://creativecommons.org/licenses/by/4.0/deed.de) veröffentlicht, welche die Nutzung, Vervielfältigung, Bearbeitung, Verbreitung und Wiedergabe in jeglichem Medium und Format erlaubt, sofern Sie den/die ursprünglichen Autor(en) und die Quelle ordnungsgemäß nennen, einen Link zur Creative Commons Lizenz beifügen und angeben, ob Änderungen vorgenommen wurden.

Die in diesem Kapitel enthaltenen Bilder und sonstiges Drittmaterial unterliegen ebenfalls der genannten Creative Commons Lizenz, sofern sich aus der Abbildungslegende nichts anderes ergibt. Sofern das betreffende Material nicht unter der genannten Creative Commons Lizenz steht und die betreffende Handlung nicht nach gesetzlichen Vorschriften erlaubt ist, ist für die oben aufgeführten Weiterverwendungen des Materials die Einwilligung des jeweiligen Rechteinhabers einzuholen.

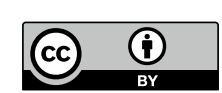

